\title{
Combined Detector of Locally-Oriented Structures and Corners in Images Based on a Scale-Space Tensor Representation of Local Neighborhoods of Pixels
}

\author{
Bogusław Cyganek \\ University of Mining and Metallurgy \\ Department of Electronics \\ Al. Mickiewicza 30, 30-059 Kraków, Poland \\ cyganek@uci.agh.edu.pl
}

\begin{abstract}
Detection of low-level image features such as edges or corners has been an essential task of image processing for many years. Similarly, detectors of such image features constitute basic building blocks of almost every image processing system. However, today's growing amount of vision applications requires at least twofold research directions: search for detectors that work better than the other, at least for a chosen group of images of interest, and - at the other hand - search for new image features, such as textons or oriented structures of local neighborhoods of pixels. In this paper we present a new approach to the old problem of corner detection, as well as detection of areas in images that can be characterized by the same angular orientation. Both detecting techniques are based on a scale-space tensor representation of local structures, and present computationally attractive image feature detectors.
\end{abstract}

\section{Introduction}

The paper addresses the problem of detection of locally-oriented structures and corners in images or sequences of images. Although, the problem of feature detection has been studied for many years and by many researchers $[5][4][9][7][8][15][16]$, there is still growing demand on computationally efficient algorithms. One of the measures of a detector efficiency is its time-memory complexity, but also or even more frequently, its robustness in respect to different scenes for real images. The latter can be stated in terms of a repeatability and detection quality in respect to the amount of thresholds that need to be defined for a given detector. Very profound overview of over six corner detectors, as well as advanced methods of their examination, was presented by Schmid and Mohr [11].

The combined method for detection of corners and locally-oriented structures, presented in this paper, belongs to a group of signal based methods. For corner detection it can be conceived as a modified version of the Harris detector 5], mostly due to the precise computation of directional derivatives. Simultaneously, this method allows for better localization of image features by restricting a detection area to contours found in images. The method can be easily applied not only for detection of 
corners, but also for detection of common pixel clusters that are characterized by the same local orientation.

The presented theoretical foundations, as well as practical implementation issues, came from the research in the field of stereo processing methods and a quest for an image feature detector that lets avoid false matches during stereo processing [3]. However, the presented concepts are by no means limited only to the stereo processing. They can be used in almost any image processing system, such as for example image and video indexing which consists of retrieval of interested points that are further used in a database search. Comparative video indexing method, based on a multiresolution contrast energy, presents paper by Bres and Jolion [2.

\section{Tensor Detector of Local Structures in Pixel Neighborhoods}

Let us analyze an image with local neighborhood $U$ defined around a point $x_{0}$ (Fig. 1 ) where each point has been additionally endowed with a directional vector, e.g. an intensity gradient.

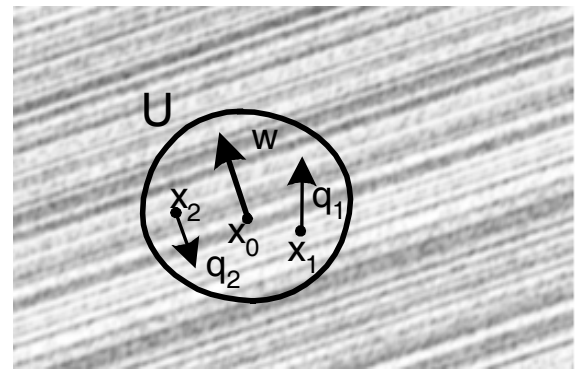

Fig. 1. Local neighborhood of a pixel $x_{0}$ with shown local gradient vectors $q_{i}$ and structural vector $w$

The goal now is to find such a vector $w$ that in a uniform way represents all other directional vectors $q_{i}$ from $U\left(x_{0}\right)$. In order to compare vectors we use their inner product.

Additionally we assume the following:

1. Direction of $w$ is invariant under rotation of $\pi$ radians;

2. Angle and module of $w$ follow signal changes in an image;

3. There is an additional measure of coherency of the local structure.

Thus, the vector $w$ at a point $x_{0}$ is an estimator of an average orientation in a neighborhood $U\left(x_{0}\right)$ that maximizes the following formula 6 :

$$
Q=\int_{U\left(x_{0}\right)}\left(q^{T}(\vec{x}) w\left(\vec{x}_{0}\right)\right)^{2} d \vec{x}
$$

The square of the inner product in (1) fulfills the invariant assumption on rotation of $\pi$ radians. Otherwise parallel and anti-parallel configurations of vectors would cancel out.

Let us introduce a symmetric tensor $\mathbf{T}$, defined as follows: 


$$
\mathbf{T}\left(\overrightarrow{x_{0}}\right)=\int_{U} \overrightarrow{\mathbf{q}}(\vec{x}) \overrightarrow{\mathbf{q}}^{T}(\vec{x}) d \vec{x}
$$

where $q(x) q^{T}(x)$ stands for an outer product of vectors and $U$ is a local neighborhood of pixels around the point $x_{0}$. Components of $\mathbf{T}$ can be described by the following formula:

$$
T_{i j}=\int_{U} q_{i}(\vec{x}) q_{j}(\vec{x}) d \vec{x} .
$$

Taking into account $(1)$ to $(3)$, the problem of finding structural vector $w$ is reduced to the solution of the maximization problem which is as follows [6:

$$
\max _{w}(Q)=\max _{w}(\vec{w} \mathbf{T} \vec{w})
$$

Expression (4) is fulfilled if $w$ is an eigenvector corresponding to the maximum eigenvalue of $\mathbf{T}$. Thus, the problem of finding structural vector $w$ reduces to the analysis of eigenvalues of $\mathbf{T}$. It can be solved analytically, as follows [3]:

$$
\lambda_{1,2}=\frac{1}{2}\left(\left(T_{x x}+T_{y y}\right) \pm \sqrt{\left(T_{x x}-T_{y y}\right)^{2}+4 T_{x y}{ }^{2}}\right),
$$

whereas trace $\operatorname{Tr}(\mathbf{T})$ of $\mathbf{T}$, which will be helpful further on during the classification of image structures, can be expressed as:

$$
\lambda_{1}+\lambda_{2}=T_{x x}+T_{y y}=\operatorname{Tr}(\mathbf{T})
$$

Based on (5), (6), and basic algebra we can try to classify local structures in images based on eigenvalues and trace of $\mathbf{T}$. Results summarizes the Table 1.

Table 1. Classification of local structures based on eigenvalues and rank of $\mathbf{T}$

\begin{tabular}{|c|l|l|}
\hline Rank of T & \multicolumn{1}{|c|}{ Eigenvalues } & \multicolumn{1}{|c|}{ Type of a local structure in an image } \\
\hline 0 & $\lambda_{1}=\lambda_{2}=0$ & Constant intensity value. \\
\hline 1 & $\lambda_{1}>0, \quad \lambda_{2}=0$ & $\begin{array}{l}\text { Ideal local orientation. The eigenvector, } \\
\text { corresponding to the eigenvalue different from zero, } \\
\text { points in a direction of maximum changes of } \\
\text { intensity. }\end{array}$ \\
\hline 2 & $\lambda_{1}>0, \quad \lambda_{2}>0$ & $\begin{array}{l}\text { Both eignevalues greater than zero mean changes in } \\
\text { all directions of a local neighborhood. The structure } \\
\text { vector can be found if one eigenvalue is dominating. }\end{array}$ \\
\hline 2 & $\lambda_{1}=\lambda_{2}>0$ & $\begin{array}{l}\text { An isotropic gray value structure. Intensity changes } \\
\text { equally in all directions. }\end{array}$ \\
\hline
\end{tabular}


Eigenvectors of $\mathbf{T}$ are formed as a columns of an adjoint matrix $[\mathbf{T}-\lambda \mathbf{1}]_{\mathrm{ad}}$, as follows:

$$
\left[\begin{array}{ll}
\mathbf{a} & \mathbf{b}
\end{array}\right]=\left[\begin{array}{ll}
a_{1} & b_{1} \\
a_{2} & b_{2}
\end{array}\right]=\left[\mathbf{T}-\lambda_{i} \mathbf{1}_{2}\right]_{a d}=\left[\begin{array}{cc}
T_{y y}-\lambda_{i} & -T_{x y} \\
-T_{x y} & T_{x x}-\lambda_{i}
\end{array}\right],
$$

where $a$ and $b$ are linearly dependent eigenvectors, $\lambda_{i}$ denotes an eigenvalue of $\mathbf{T}$. Taking into an account (5) we obtain:

$$
\left[\begin{array}{ll}
a_{1} & b_{1} \\
a_{2} & b_{2}
\end{array}\right]=\left[\begin{array}{cc}
\frac{\left(T_{y y}-T_{x x}\right) \pm \sqrt{\left(T_{x x}-T_{y y}\right)^{2}+4 T_{x y}^{2}}}{2} & -T_{x y} \\
-T_{x y} & \frac{-\left(T_{y y}-T_{x x}\right) \pm \sqrt{\left(T_{x x}-T_{y y}\right)^{2}+4 T_{x y}{ }^{2}}}{2}
\end{array}\right]
$$

A proper sign of the square root in $(8)$ is chosen based on an eigenvalue.

In order to fulfill the rotation invariant assumption, an angle of the searched structural vector must change as a doubled angle (denoted further on as $\xi$ ) of an eigenvector corresponding to the maximum eigenvalue. Thus, from the simple tangent relationship

$$
\operatorname{tg}(2 \xi)=\frac{2 \operatorname{tg}(\xi)}{1-\operatorname{tg}^{2}(\xi)} \quad, \quad \operatorname{tg}(\xi) \neq 1,
$$

and from $(8)$ we obtain easily:

$$
\begin{aligned}
& \operatorname{tg}(2 \xi)=\frac{-4 T_{x y}\left\lfloor\left(T_{y y}-T_{x x}\right) \pm \sqrt{\left(T_{x x}-T_{y y}\right)^{2}+4 T_{x y}^{2}}\right\rfloor}{\left(T_{y y}-T_{x x}\right)^{2} \pm 2\left(T_{y y}-T_{x x}\right) \sqrt{\left(T_{x x}-T_{y y}\right)^{2}+4 T_{x y}^{2}}+\left(T_{y y}-T_{x x}\right)^{2}+4 T_{x y}^{2}-4 T_{x y}^{2}}= \\
& =\frac{-4 T_{x y}\left[\left(T_{y y}-T_{x x}\right) \pm \sqrt{\left(T_{x x}-T_{y y}\right)^{2}+4 T_{x y}^{2}}\right]}{2\left(T_{y y}-T_{x x}\right)\left[\left(T_{y y}-T_{x x}\right) \pm \sqrt{\left(T_{x x}-T_{y y}\right)^{2}+4 T_{x y}^{2}}\right.}=\frac{2 T_{x y}}{T_{x x}-T_{y y}} \quad, \quad T_{x x} \neq T_{y y} .
\end{aligned}
$$

Because of the orthogonality of eigenvectors, result (10) does not depend on the choice of a particular eigenvalue in (5).

Defining an angle $\theta=2 \xi$ of a structural vector $w$

$$
\operatorname{tg}(\theta)=\operatorname{tg}(2 \xi)=\frac{2 T_{x y}}{T_{x x}-T_{y y}}=\frac{w_{2}}{w_{1}} \quad, \quad T_{x x} \neq T_{y y},
$$

we can finally discover components of $w$ which can be described as: 


$$
\mathbf{w}=\left[\begin{array}{c}
w_{1} \\
w_{2}
\end{array}\right]=\left[\begin{array}{c}
T_{x x}-T_{y y} \\
2 T_{x y}
\end{array}\right] .
$$

The structural vector (12) can now be extended by a third component which is equal to the trace of the tensor $\mathbf{T}$ :

$$
\mathbf{w}^{\prime}=\left[\begin{array}{c}
\operatorname{Tr}(\mathbf{T}) \\
\mathbf{w}
\end{array}\right]=\left[\begin{array}{c}
T_{x x}+T_{y y} \\
T_{x x}-T_{y y} \\
2 T_{x y}
\end{array}\right] .
$$

Thanks to this new component in (13) we can distinguish the case $\lambda_{1}=\lambda_{2}=0$ (constant intensity) from the case $\lambda_{1}=\overline{\lambda_{2}}>0$ (ideal isotropy).

For low-level features detection, the modified version of the structural vector $w$ ' takes the form of the vector $s$ with components defined as follows:

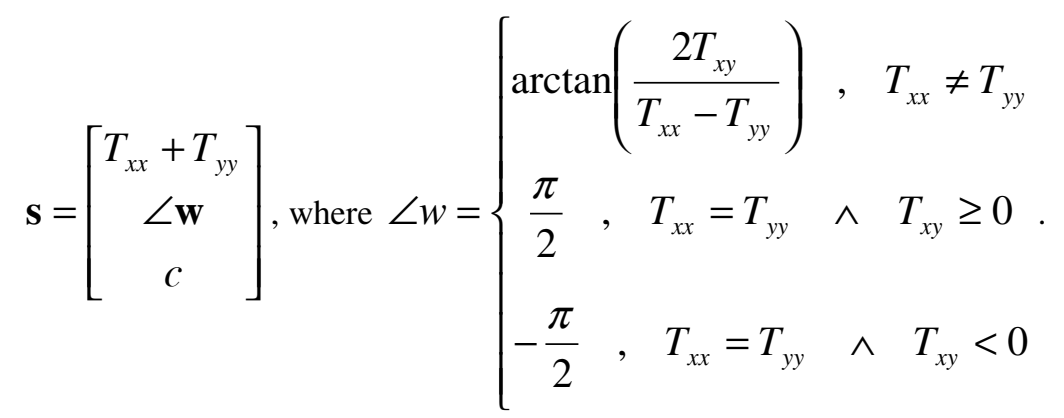

The first component in (14) is not changed from the corresponding component of $w^{\prime}$. The third component $c$ of $(14)$ constitutes a convenient coherency measure [10]:

$$
c=\left\{\begin{array}{cc}
\left(\frac{\lambda_{1}-\lambda_{2}}{\lambda_{1}+\lambda_{2}}\right)^{2}=\frac{\|\mathbf{w}\|^{2}}{(\operatorname{Tr}(\mathbf{T}))^{2}} & \operatorname{Tr}(\mathbf{T}) \neq 0 \\
0 & , \quad \operatorname{Tr}(\mathbf{T})=0
\end{array}\right.
$$

Coefficient $c$ takes on 0 for ideal isotropic areas or structures with constant intensity value, and up to 1 for ideally directional structure.

\subsection{Determining Structural Tensor for Different Scales}

Introducing some window function $h(x)$ into (3) the structural tensor components can be expressed as follows [6]: 


$$
T_{i j}=\int_{-\infty}^{+\infty} h\left(\vec{x}_{0}-\vec{x}\right) \frac{\partial I(\vec{x})}{\partial x_{i}} \frac{\partial I(\vec{x})}{\partial x_{j}} d \vec{x} .
$$

Formula (16) describes a convolution of a window function and product of directional gradients. Thus, for digital realizations (16) reduces to the following computation:

$$
\hat{T}_{i j}=F\left(R_{i} R_{j}\right),
$$

where $\hat{T}_{i j}$ stands for discrete values of the structural tensor $\mathbf{T}, F$ is a smoothing operator in a local neighborhood of pixels, $R_{k}$ is a discrete gradient operator in $k$-th direction. The required quality of the detectors is achieved in practice by application of proper discrete gradient masks $R_{k}$ in (17) - the most isotropic filter the better results. We found that the best results were obtained with matched filters such as the one proposed by Simoncelli [12, with the smoothing prefilter $p_{k}$ and differentiating filter $d_{k}$ masks in a form as follows:

$$
\begin{aligned}
& p_{5}=\left[\begin{array}{lllrl}
0.035697 & 0.248874 & 0.430855 & 0.248874 & 0.035697
\end{array}\right] \\
& d_{5}=\left[\begin{array}{lllll}
0.107663 & -0.282671 & 0 & 0.282671 & -0.107663
\end{array}\right]
\end{aligned}
$$

The formulas (3) and its implementation (17) can be extended to comprise information on different scales [13]. In this method it is accomplished by applying $F$ in a form of Gaussian smoothing kernels of different size - an idea proposed by Weickert $\|14\|[\|$. Based on (17) we obtain the general expression for the components of the structural tensor $\mathbf{T}$ at different scales:

$$
\hat{T}_{i j}(\rho)=F_{\rho}\left(R_{i} R_{j}\right),
$$

where $\rho$ denotes a scale and $F_{\rho}$ is a Gaussian kernel.

\section{Detector of Locally-Oriented Structures in Images}

The equations (14), (15), and (19) can be directly used for detection of locallyoriented structures in the sense of the tensor detector of local structures in pixel neighborhoods. For a searched pixel blobs $\Omega$ that angular orientation is in a given range $s$, based on [14], we propose the following rule for their detection:

$$
\Omega=\left\{\left(x_{i}, y_{i}\right): \mathbf{s}\left(x_{i}, y_{i}\right)=\left[\begin{array}{c}
s_{1} \\
s_{2} \\
s_{3}
\end{array}\right] \wedge\left[\begin{array}{c}
s_{1}>0 \\
\left|s_{2}-s_{\vartheta}\right|<\tau_{\vartheta} \\
s_{3}>\tau_{c}
\end{array}\right]\right\} .
$$

where $\tau_{\vartheta}$ is a threshold for detection accuracy, while $\tau_{c}$ is a threshold for coherency. In our implementation, components of the structural tensor are computed in accordance with formula (19) and with differentiating filters (18). 


\section{Corner Detector}

For detection of corners we compute eigenvalues (5) of the structural tensor which components for different scales are computed in accordance with the formula (19) and filters (18). Thus we obtain the following rule for detection of corners:

$$
\mathrm{K}=\left\{\left(x_{i}, y_{i}\right): \quad \lambda_{1}\left(x_{i}, y_{i}\right) \geq \lambda_{2}\left(x_{i}, y_{i}\right) \geq \tau_{K}\right\}
$$

where $\tau_{K}$ is a threshold for eigenvalues.

To avoid a cumbersome selection of a threshold parameter for eigenvalues, a special priority queue was developed - Fig. 2.

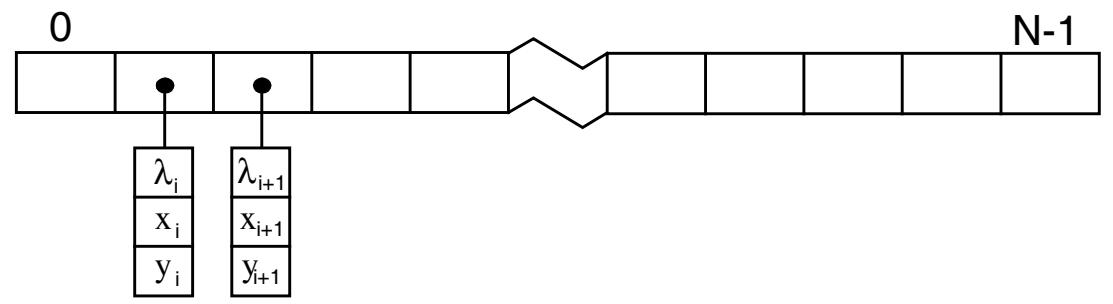

Fig. 2. Priority queue for selection of corners that comply with an assumed quality

For all elements of the queue it holds that $\lambda_{i} \leq \lambda_{i+1}$. If a new corner point is found then it can be inserted into the proper cell of the queue depending on its lowest eigenvalue. All the time the queue fulfills the constraint $\lambda_{i} \leq \lambda_{i+1}$. If a new element is inserted, then the element at index 0 is removed. This voting scheme allows for automatic selection of the best $N$ interest points.

With this data structure it is also possible to impose additional constraints, e.g. on a minimal allowable distance among adjacent corner-points. This way we can search for more "distributed" corner positions in an image. For example we can search for interest points that are at least two pixels apart.

\section{Experimental Results}

To test the presented concept we used many real and artificial images, four of them are shown in Fig. 3. Presented tests in this paper are comparative and do not check for repeatability of the detectors (that is the issue of a further research).

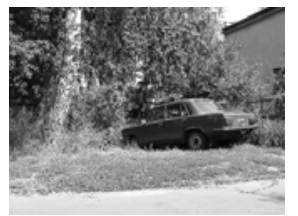

a

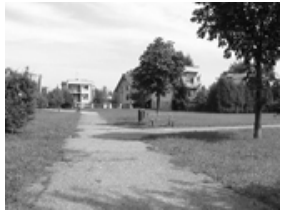

$\mathrm{b}$

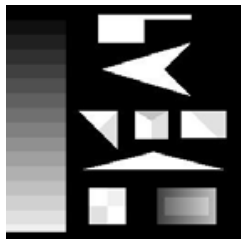

c

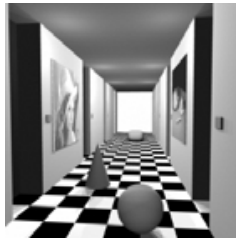

d

Fig. 3. Test images: real images (a, b), artificial images (c, d) 


\subsection{Experimental Results of Locally-Oriented Structures}

Tests for locally-oriented structures for experimental images in Fig. 3 and exemplary orientations are presented in Fig. 4.
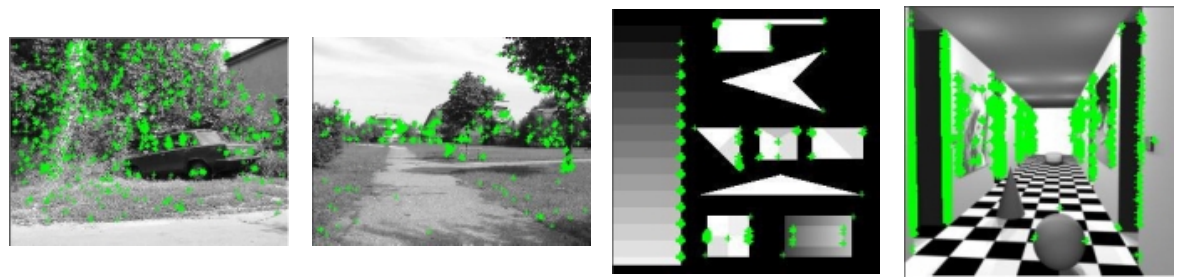

$\mathrm{a}-$ vertical orientations $\left(85-95^{\circ}\right)$
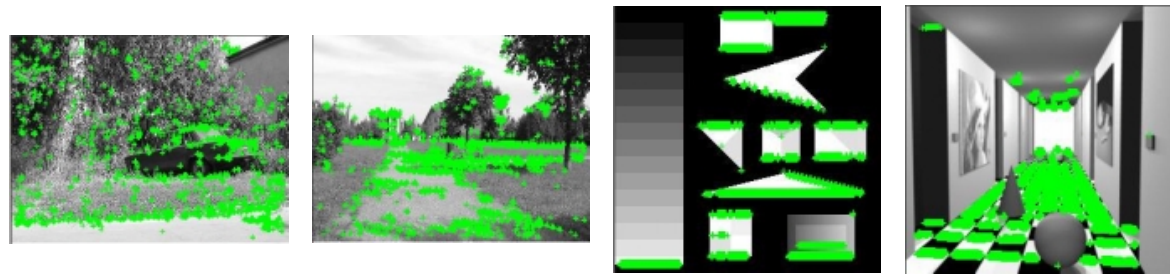

b - horizontal orientations $\left(0-10^{\circ}\right)$
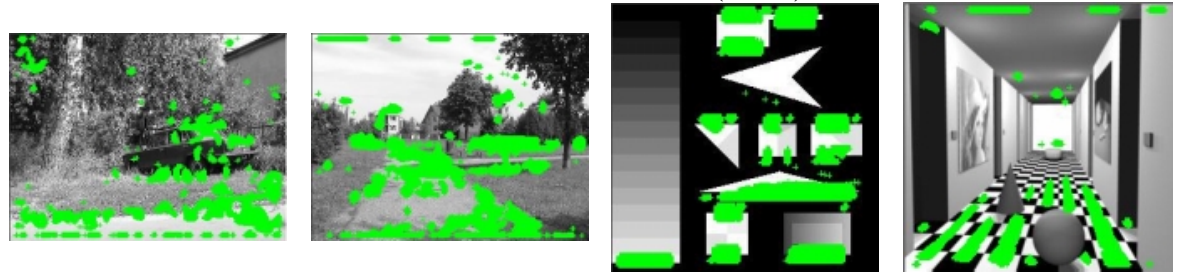

$\mathrm{c}-$ horizontal orientations $\left(0-10^{\circ}\right)$ at a coarse scale

Fig. 4. Results of the tensor detector of locally-oriented structures in test images. Found interest points are marked with a green color

The first row of Fig. 4 shows interest points that angular orientation of their local structures is in the range $85-95^{\circ}$. Most of the points have been detected correctly, although for gradually changing intensity in artificial images not all points are detected. This is due to flawed discrete gradient computation in areas of uniformly changing intensity values. Fig. $4 \mathrm{~b}$ depicts interest points with a horizontal angular orientation $\left(0-10^{\circ}\right)$, while $\mathrm{Fig}$. $4 \mathrm{c}$ shows the same computations but for different scale (Gaussian mask $13 \times 13$ ). In all experiments the threshold $\tau_{\vartheta}$ was set to 0.05 and $\tau_{c}$ to 0 .

\subsection{Experimental Results of Corner Detection}

Fig. 5 contains experimental results of the corner detector with different working parameters for test images in Fig. 3. 

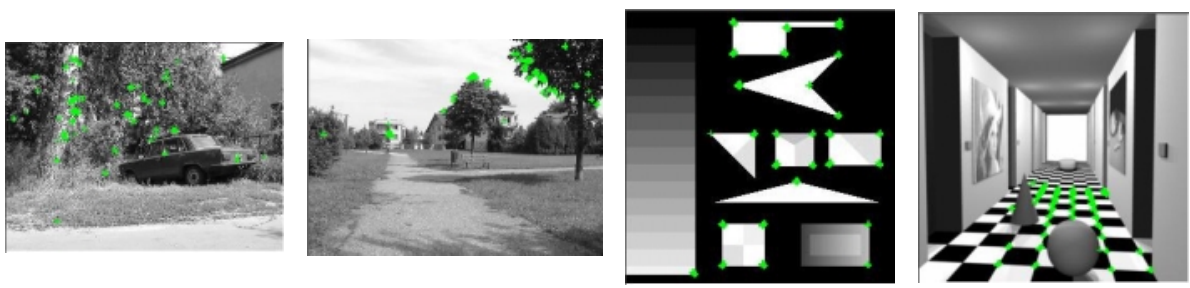

a - max. 200 corners, self distance of detected points not limited
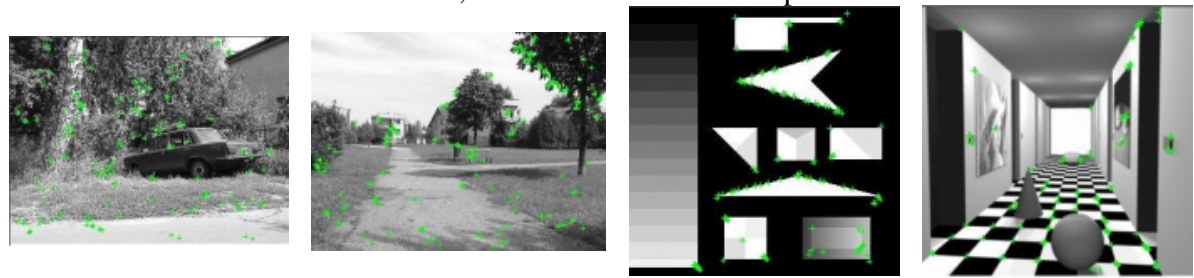

b - max. 200 corners, self distance set to be at least 1 pixel
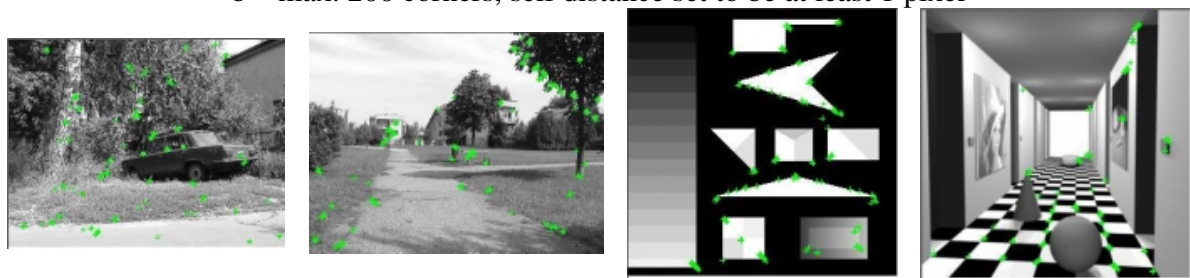

$\mathrm{c}$ - the same as (b) but at a coarse scale

Fig. 5. Results of the corner detector for test images. Found interest points are marked with a green color

Fig. 5 A depicts test images with marked corner points without any constraint on their mutual position. No threshold value was needed as well, because of the voting technique (Fig. 2). The length of the priority queue was: $\mathrm{N}=200$.

An additional constraint on pixel positions was set for experiments presented in Fig. 5p. In this case we search for the best interest points, but also it is guaranteed that the found points are at least 1 pixel from each other.Fig. 5c depicts results obtained with the same settings but at a different scale (Gaussian filter $9 \times 9$ ).

\section{Conclusions}

In this paper we have presented a novel concept of detection of interest points based on the tensor representation of local structures in images. Two types of detectors was considered: the detector of angular orientations and the corner detector. Theoretical foundations of both have been shown, as well as their practical implementations. The advantages of the detectors can be stated as follows:

- Combined detector of corners and oriented-structures.

- Precise localization due to matched directional filters.

- Scale space operations.

- No threshold for corner detection due to the voting mechanism. 
- Tensor method can be easily extended to a third dimension and then directly applied to examination of interest points, e.g. in video sequences.

At the other hand, the known drawback are as follows:

- Gradient computation cannot work properly for uniformly changed intensities.

- The presented detector has not been yet tested in regard to its repeatability in a sense stated by Schmid and Mohr [11].

Numerous experiments showed great usefulness of the presented techniques for low-level image processing. The proposed corner detector was designed especially for stereo matching. It was successively used then for computation of the epipolar geometry for real images.

\section{References}

1. Aubert, G., Kornprobst, P.: Mathematical Problems in Image Processing. Applied Mathematical Sciences Vol. 147, Springer (2002)

2. Bres, S., Jolion, J-M.: Detection of Interest Points for Image Indexation. Visual Information and Information Systems, D.P. Huijsmans et A.W. Smeulders editors, LNCS 1614, Springer, Proceeding of the third International Conference on Visual Information Systems, Amsterdam, The Netherlands (1999) 427-434

3. Cyganek, B.: Novel Stereo Matching Method That Employs Tensor Representation of Local Neighborhood in Images, Machine Graphics \& Vision, Special Issue on Stereogrammetry, Vol.10, No.3 (2001) 289-316

4. Deriche, R., Giraudon, G.: A Computational Approach for Corner and Vertex Detection. Int. Journal of Computer Vision, 10 (2) (1993) 101-124

5. Harris, C., Stephens, M.: A combined corner and edge detector, Proc. of 4th Alvey Vision Conf. (1988) 147-151

6. Haußecker, H., Jähne, B.: A Tensor Approach for Local Structure Analysis in MultiDimensional Images. Interdisciplinary Center for Scientific Computing, University of Heidelberg (1998)

7. Ji, Q., Haralick, R., M.: Corner Detection with Covariance Propagation. Technical Report, Intelligent Systems Laboratory (1997)

8. Lindeberg, T.: Edge detection and ridge detection with automatic scale selection. Computational Vision and Active Perception Laboratory. Technical report ISRN KTH/NA/P-96/06-SE (1996)

9. Smith, S.M., Brady, J.M.: SUSAN - A New Approach to Low Level Image Processing. Int. Journal of Computer Vision, 23 (1) (1997) 45-78

10. Jähne, B.: Digital Image Processing. 4th edition, Springer-Verlag, (1997)

11. Schmid, C., Mohr, R.: Comparing and Evaluating Interest Points. International Conference on Computer Vision, Bombay (1998)

12. Simoncelli, E.,P.: Design of Multi-Dimensional Derivative Filters. IEEE International Conference on Image Processing (1994)

13. Sporring, J., Nielsen, M., Florack, L., Johansen, P.: Gaussian Scale-Space Theory. Kluwer Academic Publishers (1997)

14. Weickert, J. : Anisotropic Diffusion in Image Processing. Teubner-Verlag (1998)

15. Würtz, R., Lourens, T.: Corner detection in color images by multiscale combination of end-stopped cortical cells. LNCS 1327, Proceedings of ICANN (1997) 901-906

16. Zheng, Z., Wang, H., Teoh, E.,K.: Analysis of gray level corner detection, Pattern Recognition Letters, 20 (1999) 149-162 